\title{
Gravitation and Experiment 1
}

\author{
Thibault DAMOUR
}

Institut des Hautes Etudes Scientifiques, 91440 Bures-sur-Yvette, France, and

DARC, CNRS - Observatoire de Paris, 92195 Meudon, France

\author{
Talk dedicated to R.H. Dicke and J.H. Taylor
}

\begin{abstract}
The confrontation between Einstein's gravitation theory and experimental results, notably binary pulsar data, is summarized and its significance discussed. Experiment and theory agree at the $10^{-3}$ level. All the basic structures of Einstein's theory (coupling of gravity to matter; propagation and self-interaction of the gravitational field, including in strong-field conditions) have been verified. However, some recent theoretical findings (cosmological relaxation toward zero scalar couplings) suggest that the present agreement between Einstein's theory and experiment might be naturally compatible with the existence of a long-range scalar contribution to gravity (such as the dilaton, or a moduli field of string theory). This provides a new theoretical paradigm, and new motivations for improving the experimental tests
\end{abstract}

\footnotetext{
${ }^{1}$ Talk given at Princeton's 250th Anniversary Conference on Critical Problems in Physics (Princeton, October 31 - November 2, 1996); to appear in the Proceedings to be published by Princeton University Press.
} 
of gravity. Ultra-high precision tests of the Equivalence Principle appear as the most sensitive way to look for possible long-range deviations from General Relativity: they might open a low-energy window on string-scale physics.

\section{Introduction}

Einstein's gravitation theory can be thought of as defined by two postulates. One postulate states that the action functional describing the propagation and self-interaction of the gravitational field is

$$
S_{\text {gravitation }}\left[g_{\mu \nu}\right]=\frac{c^{4}}{16 \pi G} \int \frac{d^{4} x}{c} \sqrt{g} R(g) .
$$

A second postulate states that the action functional describing the coupling of all the (fermionic and bosonic) fields describing matter and its electro-weak and strong interactions is a (minimal) deformation of the special relativistic action functional used by particle physicists (the so called "Standard Model"), obtained by replacing everywhere the flat Minkowski metric $f_{\mu \nu}=\operatorname{diag}(-1,+1,+1,+1)$ by $g_{\mu \nu}\left(x^{\lambda}\right)$ and the partial derivatives $\partial_{\mu} \equiv \partial / \partial x^{\mu}$ by $g$-covariant derivatives $\nabla_{\mu}$. [With the usual subtlety that one must also introduce a field of orthonormal frames, a "vierbein", for writing down the fermionic terms]. Schematically, one has

$$
\begin{gathered}
S_{\text {matter }}[\psi, A, H, g]=\int \frac{d^{4} x}{c} \sqrt{g} \mathcal{L}_{\text {matter }}, \\
\mathcal{L}_{\text {matter }}=-\frac{1}{4} \sum \frac{1}{g_{*}^{2}} \operatorname{tr}\left(F_{\mu \nu} F^{\mu \nu}\right)-\sum \bar{\psi} \gamma^{\mu} D_{\mu} \psi \\
-\frac{1}{2}\left|D_{\mu} H\right|^{2}-V(H)-\sum y \bar{\psi} H \psi,
\end{gathered}
$$

where $F_{\mu \nu}$ denotes the curvature of a $U(1), S U(2)$ or $S U(3)$ Yang-Mills connection $A_{\mu}, F^{\mu \nu}=g^{\mu \alpha} g^{\nu \beta} F_{\alpha \beta}, g_{*}$ being a (bare) gauge coupling constant; $D_{\mu} \equiv \nabla_{\mu}+A_{\mu} ; \psi$ denotes a fermion field (lepton or quark, coming in various flavours and three generations); $\gamma^{\mu}$ denotes four Dirac matrices such that $\gamma^{\mu} \gamma^{\nu}+\gamma^{\nu} \gamma^{\mu}=2 g^{\mu \nu} \mathbb{I}_{4}$, and $H$ denotes the Higgs doublet of scalar fields, with $y$ some (bare Yukawa) coupling constants. 
Einstein's theory of gravitation is then defined by extremizing the total action functional,

$$
S_{\text {tot }}[g, \psi, A, H]=S_{\text {gravitation }}[g]+S_{\text {matter }}[\psi, A, H, g] .
$$

Although, seen from a wider perspective, the two postulates (11) and (2) follow from the unique requirement that the gravitational interaction be mediated only by massless spin-2 excitations [四], the decomposition in two postulates is convenient for discussing the theoretical significance of various tests of General Relativity. Let us discuss in turn the experimental tests of the coupling of matter to gravity (postulate (2)), and the experimental tests of the dynamics of the gravitational field (postulate (迎). For more details and references we refer the reader to [2] or [3].

\section{Experimental tests of the coupling between matter and gravity}

The fact that the matter Lagrangian (3) depends only on a symmetric tensor $g_{\mu \nu}(x)$ and its first derivatives (i.e. the postulate of a "metric coupling" between matter and gravity) is a strong assumption (often referred to as the "equivalence principle") which has many observable consequences for the behaviour of localized test systems embedded in given, external gravitational fields. Indeed, using a theorem of Fermi and Cartan [4] (stating the existence of coordinate systems such that, along any given time-like curve, the metric components can be set to their Minkowski values, and their first derivatives made to vanish), one derives from the postulate (2) the following observable consequences:

$\mathrm{C}_{1}$ : Constancy of the "constants" : the outcome of local non-gravitational experiments, referred to local standards, depends only on the values of the coupling constants and mass scales entering the Standard Model. [In particular, the cosmological evolution of the universe at large has no influence on local experiments].

$\mathrm{C}_{2}$ : Local Lorentz invariance : local non-gravitational experiments exhibit no preferred directions in spacetime [i.e. neither spacelike ones (isotropy), nor timelike ones (boost invariance)]. 
$\mathrm{C}_{3}$ : "Principle of geodesics" and universality of free fall : small, electrically neutral, non self-gravitating bodies follow geodesics of the external spacetime $g_{\mu \nu}\left(x^{\lambda}\right)$. In particular, two test bodies dropped at the same location and with the same velocity in an external gravitational field fall in the same way, independently of their masses and compositions.

$\mathrm{C}_{4}$ : Universality of gravitational redshift : when intercompared by means of electromagnetic signals, two identically constructed clocks located at two different positions in a static external Newtonian potential $U(\mathbf{x})$ exhibit, independently of their nature and constitution, the (apparent) difference in clock rate:

$$
\frac{\tau_{1}}{\tau_{2}}=\frac{\nu_{2}}{\nu_{1}}=1+\frac{1}{c^{2}}\left[U\left(\mathbf{x}_{1}\right)-U\left(\mathbf{x}_{2}\right)\right]+O\left(\frac{1}{c^{4}}\right) .
$$

Many experiments or observations have tested the observable consequences $C_{1}-C_{4}$ and found them to hold within the experimental errors. Many sorts of data (from spectral lines in distant galaxies to a natural fission reactor phenomenon which took place at Oklo, Gabon, two billion years ago) have been used to set limits on a possible time variation of the basic coupling constants of the Standard Model. The best results concern the electromagnetic coupling, i.e. the fine-structure constant $\alpha_{\mathrm{em}}$. A recent reanalysis of the Oklo phenomenon gives a conservative upper bound [5]

$$
-6.7 \times 10^{-17} \mathrm{yr}^{-1}<\frac{\dot{\alpha}_{\mathrm{em}}}{\alpha_{\mathrm{em}}}<5.0 \times 10^{-17} \mathrm{yr}^{-1}
$$

which is much smaller than the cosmological time scale $\sim 10^{-10} \mathrm{yr}^{-1}$. It would be interesting to confirm and/or improve the limit (6) by direct laboratory measurements comparing clocks based on atomic transitions having different dependences on $\alpha_{\mathrm{em}}$. [Current atomic clock tests of the constancy of $\alpha_{\mathrm{em}}$ give the limit $\left|\dot{\alpha}_{\mathrm{em}} / \alpha_{\mathrm{em}}\right|<3.7 \times 10^{-14} \mathrm{yr}^{-1}[6]$.]

Any "isotropy of space" having a direct effect on the energy levels of atomic nuclei has been constrained to the impressive $10^{-27}$ level [7]. The universality of free fall has been verified at the $10^{-12}$ level both for laboratory bodies [8], e.g. (from the last reference in [8])

$$
\left(\frac{\Delta a}{a}\right)_{\mathrm{BeCu}}=(-1.9 \pm 2.5) \times 10^{-12}
$$


and for the gravitational accelerations of the Moon and the Earth toward the Sun [9],

$$
\left(\frac{\Delta a}{a}\right)_{\text {Moon Earth }}=(-3.2 \pm 4.6) \times 10^{-13} .
$$

The "gravitational redshift" of clock rates given by eq. (5) has been verified at the $10^{-4}$ level by comparing a hydrogen-maser clock flying on a rocket up to an altitude $\sim 10000 \mathrm{~km}$ to a similar clock on the ground 10.

In conclusion, the main observable consequences of the Einsteinian postulate (2) concerning the coupling between matter and gravity ("equivalence principle") have been verified with high precision by all experiments to date. The traditional paradigm (first put forward by Fierz [11]) is that the extremely high precision of free fall experiments $\left(10^{-12}\right.$ level $)$ strongly suggests that the coupling between matter and gravity is exactly of the "metric" form (2), but leaves open possibilities more general than eq. (1) for the spincontent and dynamics of the fields mediating the gravitational interaction. We shall provisionally adopt this paradigm to discuss the tests of the other Einsteinian postulate, eq. (代). However, we shall emphasize at the end that recent theoretical findings suggest a new paradigm.

\section{Tests of the dynamics of the gravitational field in the weak field regime}

Let us now consider the experimental tests of the dynamics of the gravitational field, defined in General Relativity by the action functional (1). Following first the traditional paradigm, it is convenient to enlarge our framework by embedding General Relativity within the class of the most natural relativistic theories of gravitation which satisfy exactly the matter-coupling tests discussed above while differing in the description of the degrees of freedom of the gravitational field. This class of theories are the metrically-coupled tensorscalar theories, first introduced by Fierz [11] in a work where he noticed that the class of non-metrically-coupled tensor-scalar theories previously introduced by Jordan [12 would generically entail unacceptably large violations of the consequence $\mathrm{C}_{1}$. [The fact that it would, by the same token, entail

even larger violations of the consequence $\mathrm{C}_{3}$ was, probably, first noticed by Dicke in subsequent work]. The metrically-coupled (or equivalence-principle 
respecting) tensor-scalar theories are defined by keeping the postulate (2), but replacing the postulate (1) by demanding that the "physical" metric $g_{\mu \nu}$ (coupled to ordinary matter) be a composite object of the form

$$
g_{\mu \nu}=A^{2}(\varphi) g_{\mu \nu}^{*}
$$

where the dynamics of the "Einstein" metric $g_{\mu \nu}^{*}$ is defined by the action functional (11) (written with the replacement $g_{\mu \nu} \rightarrow g_{\mu \nu}^{*}$ ) and where $\varphi$ is a massless scalar field. [More generally, one can consider several massless scalar fields, with an action functional of the form of a general nonlinear $\sigma$ model [13]]. In other words, the action functional describing the dynamics of the spin 2 and spin 0 degrees of freedom contained in this generalized theory of gravitation reads

$$
S_{\text {gravitational }}\left[g_{\mu \nu}^{*}, \varphi\right]=\frac{c^{4}}{16 \pi G_{*}} \int \frac{d^{4} x}{c} \sqrt{g_{*}}\left[R\left(g_{*}\right)-2 g_{*}^{\mu \nu} \partial_{\mu} \varphi \partial_{\nu} \varphi\right] .
$$

Here, $G_{*}$ denotes some bare gravitational coupling constant. This class of theories contains an arbitrary function, the "coupling function" $A(\varphi)$. When $A(\varphi)=$ const., the scalar field is not coupled to matter and one falls back (with suitable boundary conditions) on Einstein's theory. The simple, oneparameter subclass $A(\varphi)=\exp \left(\alpha_{0} \varphi\right)$ with $\alpha_{0} \in \mathbb{R}$ is the Jordan-FierzBrans-Dicke theory [11], [14], [15]. In the general case, one can define the (field-dependent) coupling strength of $\varphi$ to matter by

$$
\alpha(\varphi) \equiv \frac{\partial \ln A(\varphi)}{\partial \varphi} .
$$

It is possible to work out in detail the observable consequences of tensorscalar theories and to contrast them with the general relativistic case (see, e.g., ref. [13).

Let us now consider the experimental tests of the dynamics of the gravitational field that can be performed in the solar system. Because the planets move with slow velocities $\left(v / c \sim 10^{-4}\right)$ in a very weak gravitational potential $\left(U / c^{2} \sim(v / c)^{2} \sim 10^{-8}\right)$, solar system tests allow us only to probe the quasistatic, weak-field regime of relativistic gravity (technically described by the so-called "post-Newtonian" expansion). In the limit where one keeps only the first relativistic corrections to Newton's gravity (first post-Newtonian approximation), all solar-system gravitational experiments, interpreted within 
tensor-scalar theories, differ from Einstein's predictions only through the appearance of two "post-Einstein" parameters $\bar{\gamma}$ and $\bar{\beta}$ (related to the usually considered Eddington parameters $\gamma$ and $\beta$ through $\bar{\gamma} \equiv \gamma-1, \bar{\beta} \equiv \beta-1$ ). The parameters $\bar{\gamma}$ and $\bar{\beta}$ vanish in General Relativity, and are given in tensorscalar theories by

$$
\begin{gathered}
\bar{\gamma}=-2 \frac{\alpha_{0}^{2}}{1+\alpha_{0}^{2}}, \\
\bar{\beta}=+\frac{1}{2} \frac{\beta_{0} \alpha_{0}^{2}}{\left(1+\alpha_{0}^{2}\right)^{2}},
\end{gathered}
$$

where $\alpha_{0} \equiv \alpha\left(\varphi_{0}\right), \beta_{0} \equiv \partial \alpha\left(\varphi_{0}\right) / \partial \varphi_{0} ; \varphi_{0}$ denoting the cosmologicallydetermined value of the scalar field far away from the solar system. Essentially, the parameter $\bar{\gamma}$ depends only on the linearized structure of the gravitational theory (and is a direct measure of its field content, i.e. whether it is pure spin 2 or contains an admixture of spin 0 ), while the parameter $\bar{\beta}$ parametrizes some of the quadratic nonlinearities in the field equations (cubic vertex of the gravitational field).

All currently performed gravitational experiments in the solar system, including perihelion advances of planetary orbits, the bending and delay of electromagnetic signals passing near the Sun, and very accurate range data to the Moon obtained by laser echoes, are compatible with the general relativistic predictions $\bar{\gamma}=0=\bar{\beta}$ and give upper bounds on both $|\bar{\gamma}|$ and $|\bar{\beta}|$ (i.e. on possible fractional deviations from General Relativity) of order $10^{-3}$. More precisely: (i) the Viking mission measurement of the gravitational time delay of radar signals passing near the Sun ("Shapiro effect" [16]) gave [17]

$$
|\bar{\gamma}|<2 \times 10^{-3}
$$

with similar limits coming from VLBI measurements of the deflection of radio waves by the Sun [18]; (ii) the Lunar Laser Ranging measurements of a possible polarization of the orbit of the Moon toward the Sun ("Nordtvedt effect" [19]) give [9]

$$
4 \bar{\beta}-\bar{\gamma}=-0.0007 \pm 0.0010,
$$

which, combined with the above constraint on $\bar{\gamma}$, gives

$$
|\bar{\beta}|<6 \times 10^{-4}
$$


and (iii) measurement of Mercury's orbit through planetary radar ranging gave 20

$$
|\bar{\beta}|<3 \times 10^{-3},
$$

when assuming the above Viking limit on $\bar{\gamma}$ and a value of the Sun's quadrupole moment $J_{2} \sim 2 \times 10^{-7}$.

Recently, the parametrization of the weak-field deviations between generic tensor-multi-scalar theories and Einstein's theory has been extended to the second post-Newtonian order 21]. Only two post-post-Einstein parameters, $\varepsilon$ and $\zeta$, representing a deeper layer of structure of the gravitational interaction, show up. These parameters have been shown to be already significantly constrained by binary-pulsar data: $|\varepsilon|<7 \times 10^{-2},|\zeta|<6 \times 10^{-3}$. See [21] for a detailed discussion, including the consequences for the interpretation of future, higher-precision solar-system tests.

\section{Tests of the dynamics of the gravitational field in the strong field regime}

In spite of the diversity, number and often high precision of solar system tests, they have an important qualitative weakness : they probe neither the radiation properties nor the strong-field aspects of relativistic gravity. Fortunately, the discovery [22] and continuous observational study of pulsars in gravitationally bound binary orbits has opened up an entirely new testing ground for relativistic gravity, giving us an experimental handle on the regime of strong and/or radiative gravitational fields.

The fact that binary pulsar data allow one to probe the propagation properties of the gravitational field is well known. This comes directly from the fact that the finite velocity of propagation of the gravitational interaction between the pulsar and its companion generates damping-like terms in the equations of motion, i.e. terms which are directed against the velocities. [This can be understood heuristically by considering that the finite velocity of propagation must cause the gravitational force on the pulsar to make an angle with the instantaneous position of the companion [23], and was verified by a careful derivation of the general relativistic equations of motion of binary systems of compact objects [24]]. These damping forces cause the binary orbit to shrink and its orbital period $P_{b}$ to decrease. The remarkable 
stability of the pulsar clock, together with the cleanliness of the binary pulsar system, has allowed Taylor and collaborators to measure the secular orbital period decay $\dot{P}_{b} \equiv d P_{b} / d t[25]$, thereby giving us a direct experimental probe of the damping terms present in the equations of motion. Note that, contrary to what is commonly stated, the link between the observed quantity $\dot{P}_{b}$ and the propagation properties of the gravitational interaction is quite direct. [It appears indirect only when one goes through the common but unnecessary detour of a heuristic reasoning based on the consideration of the energy lost into gravitational waves emitted at infinity].

The fact that binary pulsar data allow one to probe strong-field aspects of relativistic gravity is less well known. The a priori reason for saying that they should is that the surface gravitational potential of a neutron star $G m / c^{2} R \simeq 0.2$ is a mere factor 2.5 below the black hole limit (and a factor $\sim 10^{8}$ above the surface potential of the Earth). Due to the peculiar "effacement" properties of strong-field effects taking place in General Relativity [24], the fact that pulsar data probe the strong-gravitational-field regime can only be seen when contrasting Einstein's theory with more general theories. In particular, it has been found in tensor-scalar theories [26] that a self-gravity as strong as that of a neutron star can naturally (i.e. without fine tuning of parameters) induce order-unity deviations from general relativistic predictions in the orbital dynamics of a binary pulsar thanks to the existence of nonperturbative strong-field effects. [The adjective "nonperturbative" refers here to the fact that this phenomenon is nonanalytic in the coupling strength of the scalar field, eq. (11), which can be as small as wished in the weak-field limit]. As far as we know, this is the first example where large deviations from General Relativity, induced by strong self-gravity effects, occur in a theory which contains only positive energy excitations and whose post-Newtonian limit can be arbitrarily close to that of General Relativity. [The strong-field deviations considered in previous studies [2], [13 arose in theories containing negative energy excitations.]

A comprehensive account of the use of binary pulsars as laboratories for testing strong-field gravity will be found in ref. [27]. Two complementary approaches can be pursued : a phenomenological one ("Parametrized PostKeplerian" formalism), or a theory-dependent one [13], 27].

The phenomenological analysis of binary pulsar timing data consists in fitting the observed sequence of pulse arrival times to the generic DD timing formula 28] whose functional form has been shown to be common to the whole 
class of tensor-multi-scalar theories. The least-squares fit between the timing data and the parameter-dependent DD timing formula allows one to measure, besides some "Keplerian" parameters ("orbital period" $P_{b}$, "eccentricity" $e, \ldots)$, a maximum of eight "post-Keplerian" parameters: $k, \gamma, \dot{P}_{b}, r, s, \delta_{\theta}, \dot{e}$ and $\dot{x}$. Here, $k \equiv \dot{\omega} P_{b} / 2 \pi$ is the fractional periastron advance per orbit, $\gamma$ a time dilation parameter (not to be confused with its post-Newtonian namesake), $\dot{P}_{b}$ the orbital period derivative mentioned above, and $r$ and $s$ the "range" and "shape" parameters of the gravitational time delay caused by the companion. The important point is that the post-Keplerian parameters can be measured without assuming any specific theory of gravity. Now, each specific relativistic theory of gravity predicts that, for instance, $k, \gamma, \dot{P}_{b}, r$ and $s$ (to quote parameters that have been successfully measured from some binary pulsar data) are some theory-dependent functions of the (unknown) masses $m_{1}, m_{2}$ of the pulsar and its companion. Therefore, in our example, the five simultaneous phenomenological measurements of $k, \gamma, \dot{P}_{b}, r$ and $s$ determine, for each given theory, five corresponding theory-dependent curves in the $m_{1}-m_{2}$ plane (through the 5 equations $k^{\text {measured }}=k^{\text {theory }}\left(m_{1}, m_{2}\right)$, etc...). This yields three $(3=5-2)$ tests of the specified theory, according to whether the five curves meet at one point in the mass plane, as they should. In the most general (and optimistic) case, discussed in [27], one can phenomenologically analyze both timing data and pulse-structure data (pulse shape and polarization) to extract up to nineteen post-Keplerian parameters. Simultaneous measurement of these 19 parameters in one binary pulsar system would yield 15 tests of relativistic gravity (here one must subtract 4 because, besides the two unknown masses $m_{1}, m_{2}$, generic post-Keplerian parameters can depend upon the two unknown Euler angles determining the direction of the spin of the pulsar). The theoretical significance of these tests depends upon the physics lying behind the post-Keplerian parameters involved in the tests. For instance, as we said above, a test involving $\dot{P}_{b}$ probes the propagation (and helicity) properties of the gravitational interaction. But a test involving, say, $k, \gamma, r$ or $s$ probes (as shown by combining the results of [13] and [26]) strong self-gravity effects independently of radiative effects.

Besides the phenomenological analysis of binary pulsar data, one can also adopt a theory-dependent methodology [13], [27]. The idea here is to work from the start within a certain finite-dimensional "space of theories", i.e. within a specific class of gravitational theories labelled by some the- 
ory parameters. Then by fitting the raw pulsar data to the predictions of the considered class of theories, one can determine which regions of theoryspace are compatible (at say the $90 \%$ confidence level) with the available experimental data. This method can be viewed as a strong-field generalization of the parametrized post-Newtonian formalism [2] used to analyze solar-system experiments. In fact, under the assumption that strong-gravity effects in neutron stars can be expanded in powers of the "compactness" $c_{A} \equiv-2 \partial \ln m_{A} / \partial \ln G \sim G m_{A} / c^{2} R_{A}$, Ref. [13] has shown that the observable predictions of generic tensor-multi-scalar theories could be parametrized by a sequence of "theory parameters",

$$
\bar{\gamma}, \bar{\beta}, \beta_{2}, \beta^{\prime}, \beta^{\prime \prime}, \beta_{3},\left(\beta \beta^{\prime}\right) \ldots
$$

representing deeper and deeper layers of structure of the relativistic gravitational interaction beyond the first-order post-Newtonian level parametrized by $\bar{\gamma}$ and $\bar{\beta}$ (the second layer $\beta_{2}, \beta^{\prime}$ being equivalent to the parameters $\zeta, \varepsilon$ describing the second-order post-Newtonian level [21], etc...).

When non-perturbative strong-field effects develop, one cannot use the multi-parameter approach just mentioned, based on expansions in powers of the "compactnesses". A useful alternative approach is then to work within specific, low-dimensional "mini-spaces of theories". Of particular interest is the two-dimensional mini-space of tensor-scalar theories defined by the coupling function $A(\varphi)=\exp \left(\alpha_{0} \varphi+\frac{1}{2} \beta_{0} \varphi^{2}\right)$. The predictions of this family of theories (parametrized by $\alpha_{0}$ and $\beta_{0}$ ) are analytically described, in weakfield contexts, by the post-Einstein parameter (12), and can be studied in strong-field contexts by combining analytical and numerical methods [29.

After having reviewed the theory of pulsar tests, let us briefly summarize the current experimental situation. Concerning the first discovered binary pulsar PSR1913 + 16 [22], it has been possible to measure with accuracy the three post-Keplerian parameters $k, \gamma$ and $\dot{P}_{b}$. From what was said above, these three simultaneous measurements yield one test of gravitation theories. After subtracting a small $\left(\sim 10^{-14}\right.$ level in $\left.\dot{P}_{b} !\right)$, but significant, perturbing effect caused by the Galaxy [30], one finds that General Relativity passes this $\left(k-\gamma-\dot{P}_{b}\right)_{1913+16}$ test with complete success at the $10^{-3}$ level. More precisely, one finds [31], [25]

$$
\left[\frac{\dot{P}_{b}^{\text {obs }}-\dot{P}_{b}^{\text {galactic }}}{\dot{P}_{b}^{\text {GR }}\left[k^{\text {obs }}, \gamma^{\text {obs }}\right]}\right]_{1913+16}=1.0032 \pm 0.0023(\text { obs }) \pm 0.0026(\text { galactic })
$$




$$
=1.0032 \pm 0.0035
$$

where $\dot{P}_{b}^{\mathrm{GR}}\left[k^{\mathrm{obs}}, \gamma^{\mathrm{obs}}\right]$ is the GR prediction for the orbital period decay computed from the observed values of the other two post-Keplerian parameters $k$ and $\gamma$. [More explicitly, this means that the two measurements $k^{\text {obs }}$ and $\gamma^{\text {obs }}$ are used, together with the corresponding general relativistic predictions $k^{\mathrm{obs}}=k^{\mathrm{GR}}\left(m_{1}, m_{2}\right), \gamma^{\mathrm{obs}}=\gamma^{\mathrm{GR}}\left(m_{1}, m_{2}\right)$, to compute the two masses $m_{1}$ and $m_{2}$ that enter the theoretical prediction for $\dot{P}_{b}$.]

This beautiful confirmation of General Relativity is an embarrassment of riches in that it probes, at the same time, the propagation and strong-field properties of relativistic gravity ! If the timing accuracy of PSR1913 + 16 could improve by a significant factor two more post-Keplerian parameters $(r$ and $s$ ) would become measurable and would allow one to probe separately the propagation and strong-field aspects 31. Fortunately, the discovery of the binary pulsar PSR1534 + 12 [32 (which is significantly stronger than PSR1913 + 16 and has a more favourably oriented orbit) has opened a new testing ground, in which it has been possible to probe strong-field gravity independently of radiative effects. A phenomenological analysis of the timing data of PSR1534 + 12 has allowed one to measure the four post-Keplerian parameters $k, \gamma, r$ and $s$ [31]. From what was said above, these four simultaneous measurements yield two tests of strong-field gravity, without mixing of radiative effects. General Relativity is found to pass these tests with complete success within the measurement accuracy [31], 25]. The most precise of these new, pure strong-field tests is the one obtained by combining the measurements of $k, \gamma$ and $s$. Using the data reported in [33] (with, following [21], doubled statistical uncertainties to take care of systematic errors) one finds agreement at the $1 \%$ level:

$$
\left[\frac{s^{\mathrm{obs}}}{s^{\mathrm{GR}}\left[k^{\mathrm{obs}}, \gamma^{\mathrm{obs}}\right]}\right]_{1534+12}=1.010 \pm 0.008 \text {. }
$$

More recently, it has been possible to extract also the "radiative" parameter $\dot{P}_{b}$ from the timing data of PSR $1534+12$. Again, General Relativity is found to be fully consistent (at the current $\sim 20 \%$ level) with the additional test provided by the $\dot{P}_{b}$ measurement [35], [33]. Note that this gives our second direct experimental confirmation that the gravitational interaction propagates as predicted by Einstein's theory. Moreover, an analysis of the pulse shape of PSR1534 +12 has shown that the misalignment between the 
spin vector of the pulsar and the orbital angular momentum was greater than

$8^{\circ}$ [27. This opens the possibility that this system will soon allow one to test the spin precession induced by gravitational spin-orbit coupling.

To end this brief summary, let us mention that several other binary pulsar systems (of a different class than that of $1913+16$ and $1534+12$ ) can also be used to test relativistic gravity. We have here in mind nearly circular systems made of a neutron star and a white dwarf. Such dissymetric systems are useful probes of the possible existence of dipolar gravitational waves [36] and/or of a possible violation of the universality of free fall linked to the strong self-gravity of the neutron star [37]. A theory-dependent analysis of the published pulsar data on PSRs $1913+16,1534+12$ and $0655+64$ (a dissymetric system constraining the existence of dipolar radiation) has been recently performed within the $\left(\alpha_{0}, \beta_{0}\right)$-space of tensor-scalar theories introduced above [29]. This analysis proves that binary-pulsar data exclude large regions of theory-space which are compatible with solar-system experiments. This is illustrated in Fig. 1 above (reproduced from Fig. 9 of Ref. [29]) which shows that $\beta_{0}$ must be larger than about -5 , while any value of $\beta_{0}$ is compatible with weak-field tests as long as $\alpha_{0}$ is small enough. Note that Fig. 1 is drawn in the framework of tensor-scalar theories respecting the equivalence principle. In the more general (and more plausible; see below) framework of theories where the scalar couplings violate the equivalence principle one gets much stronger constraints on the coupling parameter $\alpha_{0}$ of order $\alpha_{0}^{2} \lesssim 10^{-7}$ [34].

For a general review of the use of pulsars as physics laboratories the reader can consult Ref. 39].

\section{$5 \quad$ Was Einstein $100 \%$ right ?}

Summarizing the experimental evidence discussed above, we can say that Einstein's postulate of a pure metric coupling between matter and gravity ("equivalence principle") appears to be, at least, $99.9999999999 \%$ right (because of universality-of-free-fall experiments), while Einstein's postulate (1) for the field content and dynamics of the gravitational field appears to be, at least, $99.9 \%$ correct both in the quasi-static-weak-field limit appropriate to solar-system experiments, and in the radiative-strong-field regime explored by binary pulsar experiments. Should one apply Occam's razor and decide 


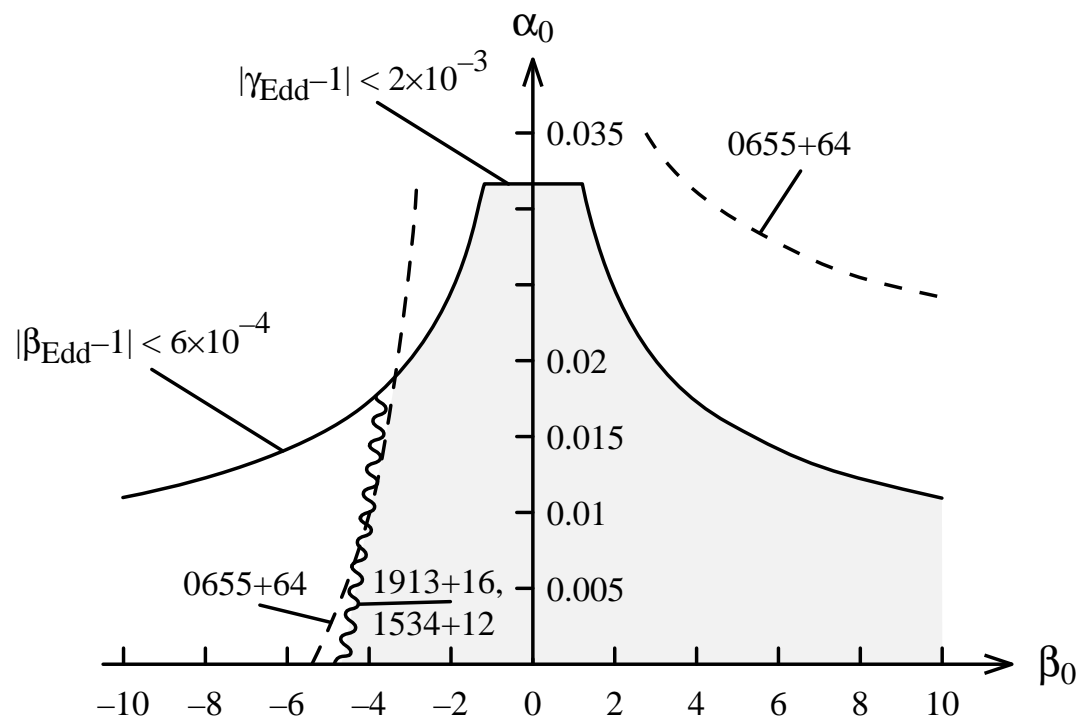

Figure 1: Regions of the $\left(\alpha_{0}, \beta_{0}\right)$-plane allowed by (composition-independent) solar-system experiments and three binary pulsar experiments. The region simultaneously allowed by all the tests is shaded. Note that binary pulsar tests exclude a large portion of the region (below the solid line) allowed by solar-system tests. (Figure taken from Ref. [29.)

that Einstein must have been $100 \%$ right, and then stop testing General Relativity? My answer is definitely, no !

First, one should continue testing a basic physical theory such as General Relativity to the utmost precision available simply because it is one of the essential pillars of the framework of physics. This is the fundamental justification of an experiment such as Gravity Probe B (the Stanford gyroscope experiment), which will advance by two orders of magnitude our experimental knowledge of post-Newtonian gravity.

Second, some very crucial qualitative features of General Relativity have not yet been verified : in particular the existence of black holes, and the direct detection on Earth of gravitational waves. Hopefully, the LIGO/VIRGO network of interferometric detectors will observe gravitational waves early in the next century. [See the contribution of Kip Thorne to these proceedings.]

Last, some recent theoretical findings suggest that the current level of precision of the experimental tests of gravity might be naturally (i.e. without 
fine tuning of parameters) compatible with Einstein being actually only $50 \%$ right ! By this we mean that the correct theory of gravity could involve, on the same fundamental level as the Einsteinian tensor field $g_{\mu \nu}^{*}$, a massless scalar field $\varphi$.

Let us first question the traditional paradigm (initiated by Fierz [11 and enshrined by Dicke [15], Nordtvedt and Will [2]) according to which special attention should be given to tensor-scalar theories respecting the equivalence principle. This class of theories was, in fact, introduced in a purely ad hoc way so as to prevent too violent a contradiction with experiment. However, it is important to notice that the scalar couplings which arise naturally in theories unifying gravity with the other interactions systematically violate the equivalence principle. This is true both in Kaluza-Klein theories (which were the starting point of Jordan's theory) and in string theories. In particular, it is striking that (as first noted by Scherk and Schwarz [40]) the dilaton field $\Phi$, which plays an essential role in string theory, appears as a necessary partner of the graviton field $g_{\mu \nu}$ in all string models. Let us recall that $g_{s}=e^{\Phi}$ is the basic string coupling constant (measuring the weight of successive string loop contributions) which determines, together with other scalar fields (the moduli), the values of all the coupling constants of the low-energy world. This means, for instance, that the fine-structure constant $\alpha_{\mathrm{em}}$ is a function of $\Phi$ (and possibly of other moduli fields). This spatiotemporal variability of coupling constants entails a clear violation of the equivalence principle. In particular, $\alpha_{\mathrm{em}}$ would be expected to vary on the Hubble time scale (in contradiction with the limit (6) above), and materials of different compositions would be expected to fall with different accelerations (in contradiction with the limits (7), (8) above).

The most popular idea for reconciling gravitational experiments with the existence, at a fundamental level, of scalar partners of $g_{\mu \nu}$ is to assume that all these scalar fields (which are massless before supersymmetry breaking) will acquire a mass after supersymmetry breaking. Typically one expects this mass $m$ to be in the TeV range [41]. This would ensure that scalar exchange brings only negligible, exponentially small corrections $\propto \exp (-m r / \hbar c)$ to the general relativistic predictions concerning low-energy gravitational effects.

However, this idea is fraught with many cosmological difficulties. A first difficulty is that, the dilaton being protected from getting a mass to all orders of perturbation theory, any putative non-perturbative potential $V(\Phi)$ will be extremely shallow, which makes it difficult to fix the VEV of $\Phi$ without 
fine-tuning the initial conditions [42]. A second difficulty is that additional fine-tuning (or some new mechanism) is needed to ensure that the value of the potential $V(\Phi)$ at its minimum is zero, or at least 120 orders of magnitude smaller than the Planck density ("cosmological constant problem"). A third problem is that one generically expects a lot of potential energy to be stored initially in $V(\Phi)$. The cosmological decay of this energy is either too slow or leads to an overproduction of entropy ("Polonyi problem" [43]). Moreover, if cosmological strings exist they tend to radiate a lot of dilatons thereby causing a problem similar to the usual Polonyi problem [44].

Though these cosmological difficulties might be solved by a combination of ad hoc solutions (e.g. introducing a secondary stage of inflation to dilute previously produced dilatons [45], [46]), a more radical solution to the problem of reconciling the existence of the dilaton (or any moduli field) with experimental tests and cosmological data has been proposed [47] (see also [48] which considered an equivalence-principle-respecting scalar field). The main idea of Ref. [47] is that string-loop effects (i.e. corrections depending upon $g_{s}=e^{\Phi}$ induced by worldsheets of arbitrary genus in intermediate string states) may modify the low-energy, Kaluza-Klein type matter couplings $\left(\propto e^{-2 \Phi} F_{\mu \nu} F^{\mu \nu}\right)$ of the dilaton (or moduli) in such a manner that the VEV of $\Phi$ be cosmologically driven toward a finite value $\Phi_{m}$ where it decouples from matter. For such a "least coupling principle" to hold, the loop-modified coupling functions of the dilaton, $B_{i}(\Phi)=e^{-2 \Phi}+c_{0}+c_{1} e^{2 \Phi}+\cdots+$ (nonperturbative terms), must exhibit extrema for finite values of $\Phi$, and these extrema must have certain universality properties. More precisely, the most general low-energy couplings induced by string-loop effects will be such that the various terms on the right-hand side of eq. (3) will be multiplied by several different functions of the scalar field(s) : say a factor $B_{F}(\varphi)$ in factor of the kinetic terms of the gauge fields, a factor $B_{\psi}(\varphi)$ in factor of the Dirac kinetic terms, etc... We work here in the Einstein frame, and with a canonically normalized scalar field $\varphi$, i.e. the Lagrangian density has the form

$$
\mathcal{L}=\frac{1}{16 \pi G_{*}}\left[R\left(g_{*}\right)-2 g_{*}^{\mu \nu} \partial_{\mu} \varphi \partial_{\nu} \varphi\right]-\frac{1}{4} B_{F}(\varphi) F_{\mu \nu} F^{\mu \nu}+\cdots
$$

It has been shown in 477 that if the various coupling functions $B_{i}(\varphi), i=$ $F, \psi, \ldots$, all admit an extremum (which must be a maximum for the "leading" $B_{i}$ ) at some common value $\varphi_{m}$ of $\varphi$, the cosmological evolution of the coupled 
tensor-scalar-matter system will drive $\varphi$ towards the value $\varphi_{m}$, at which $\varphi$ decouples from matter. As suggested in 47 a natural way in which the required conditions could be satisfied is through the existence of a discrete symmetry in scalar space. [For instance, a symmetry under $\varphi \rightarrow-\varphi$ would guarantee that all the scalar coupling functions reach an extremum at the self-dual point $\left.\varphi_{m}=0\right]$. The existence of such symmetries have been proven for some of the scalar fields appearing in string theory (target-space duality for the moduli fields) and conjectured for others ( $S$-duality for the dilaton). This gives us some hope that the mechanism of 477 could apply and thereby naturally reconcile the existence of massless scalar fields with experiment.

A study of the efficiency of attraction of $\varphi$ towards $\varphi_{m}$ estimates that the present vacuum expectation value $\varphi_{0}$ of the scalar field would differ (in a rms sense) from $\varphi_{m}$ by

$$
\varphi_{0}-\varphi_{m} \sim 2.75 \times 10^{-9} \times \kappa^{-3} \Omega^{-3 / 4} \Delta \varphi
$$

where $\kappa$ denotes the curvature of $\ln B_{F}(\varphi)$ around the maximum $\varphi_{m}$ and $\Delta \varphi$ the deviation $\varphi-\varphi_{m}$ at the beginning of the (classical) radiation era. Equation (22) predicts (when $\Delta \varphi$ is of order unity [2) the existence, at the present cosmological epoch, of many small, but not unmeasurably small, deviations from General Relativity proportional to the square of $\varphi_{0}-\varphi_{m}$. This provides a new incentive for trying to improve by several orders of magnitude the various experimental tests of Einstein's equivalence principle, i.e. of the consequences $C_{1}-C_{4}$ recalled above. The most sensitive way to look for a small residual violation of the equivalence principle is to perform improved tests of the universality of free fall. The mechanism of Ref. [47 suggests a specific composition-dependence of the residual differential acceleration of free fall and estimates that a non-zero signal could exist at a very small level as illustrated in Fig. 2 (taken from Ref. [47). The dashed line in this Figure is (as Eq. (22) above) a rough analytical estimate (assuming random phases) which reads

$$
\left(\frac{\Delta a}{a}\right)_{\mathrm{rms}}^{\max } \sim 1.36 \times 10^{-18} \kappa^{-4} \Omega^{-3 / 2}(\Delta \varphi)^{2}
$$

where $\kappa$ is expected to be of order unity (or smaller, leading to a larger signal, in the case where $\varphi$ is a modulus rather than the dilaton).

\footnotetext{
${ }^{2}$ However, $\Delta \varphi$ could be $\ll 1$ if the attractor mechanism already applies during an early stage of potential-driven inflation 44.
} 


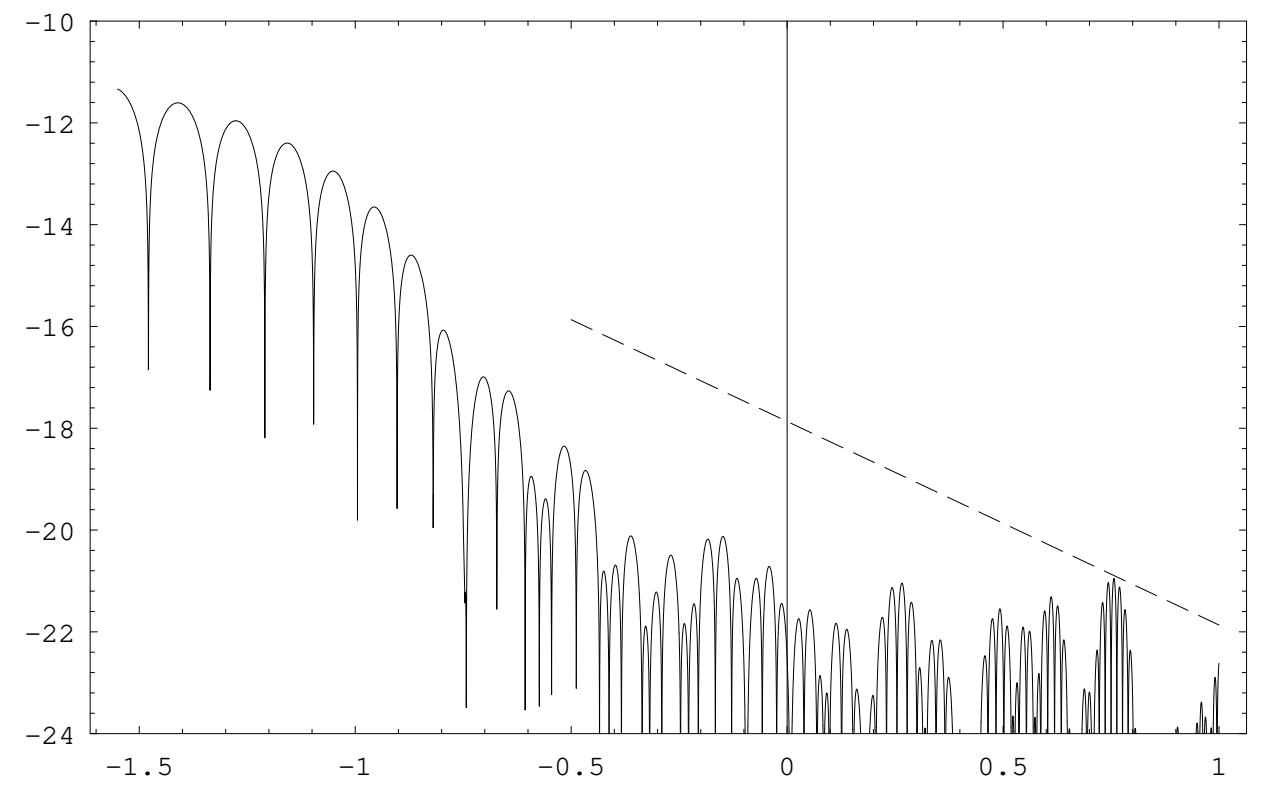

Figure 2: The solid line represents $\log _{10}(\Delta a / a)_{\max }$ as a function of $\log _{10} \kappa$, i.e. the expected present level of violation of the universality of free fall as a function of the curvature $\kappa$ of the (string-loop induced) coupling function $\ln B_{F}^{-1}(\varphi)$ near a minimum $\varphi$. The dashed line is a rough analytical estimate (assuming random phases of oscillations). (Figure taken from Ref. [47].)

Let us emphasize again that the strength of the cosmological scenario considered here as counterargument to applying Occam's razor lies in the fact that the very small number on the right-hand side of eq. (23) has been derived without any fine tuning or use of small parameters, and turns out to be naturally smaller than the $10^{-12}$ level presently tested by equivalenceprinciple experiments (see equations (7), (8)). The estimate (23) gives added significance to the project of a Satellite Test of the Equivalence Principle (nicknamed STEP, and currently studied by NASA, ESA and CNES) which aims at probing the universality of free fall of pairs of test masses orbiting the Earth at the $10^{-17}$ or $10^{-18}$ level [50].

\section{References}


[1] R.P. Feynman, F.B. Morinigo and W.G. Wagner, Feynman Lectures on Gravitation, edited by Brian Hatfield (Addison-Wesley, Reading, 1995);

S. Weinberg, Phys. Rev. 138 (1965) B988,

V.I. Ogievetsky and I.V. Polubarinov, Ann. Phys. N.Y. 35 (1965) 167 ;

W. Wyss, Helv. Phys. Acta 38 (1965) 469;

S. Deser, Gen. Rel. Grav. 1 (1970) 9;

D.G. Boulware and S. Deser, Ann. Phys. N.Y. 89 (1975) 193;

J. Fang and C. Fronsdal, J. Math. Phys. 20 (1979) 2264;

R.M. Wald, Phys. Rev. D 33 (1986) 3613;

C. Cutler and R.M. Wald, Class. Quantum Grav. 4 (1987) 1267;

R.M. Wald, Class. Quantum Grav. 4 (1987) 1279.

[2] C.M. Will, Theory and Experiment in Gravitational Physics, 2nd edition (Cambridge University Press, Cambridge, 1993); and Int. J. Mod. Phys. D 1 (1992) 13.

[3] T. Damour, Gravitation and Experiment in Gravitation and Quantizations, eds B. Julia and J. Zinn-Justin, Les Houches, Session LVII (Elsevier, Amsterdam, 1995), pp 1-61.

[4] E. Fermi, Atti Accad. Naz. Lincei Cl. Sci. Fis. Mat. \& Nat. 31 (1922) 184 and 306;

E. Cartan, Leçons sur la Géométrie des Espaces de Riemann (Gauthier-Villars, Paris, 1963).

[5] T. Damour and F. Dyson, Nucl. Phys. B 480 (1996) 37; hep$\mathrm{ph} / 9606486$.

[6] J.D. Prestage, R.L. Tjoelker and L. Maleki, Phys. Rev. Lett. 74 (1995) 3511.

[7] J.D. Prestage et al., Phys. Rev. Lett. 54 (1985) 2387;

S.K. Lamoreaux et al., Phys. Rev. Lett. 57 (1986) 3125;

T.E. Chupp et al., Phys. Rev. Lett. 63 (1989) 1541. 
[8] P.G. Roll, R. Krotkov and R.H. Dicke, Ann. Phys. (N.Y.) 26 (1964) 442;

V.B. Braginsky and V.I. Panov, Sov. Phys. JETP 34 (1972) 463;

Y. Su et al., Phys. Rev. D 50 (1994) 3614.

[9] J.O. Dickey et al., Science 265 (1994) 482; J.G. Williams, X.X. Newhall and J.O. Dickey, Phys. Rev. D 53 (1996) 6730.

[10] R.F.C. Vessot and M.W. Levine, Gen. Rel. Grav. 10 (1978) 181;

R.F.C. Vessot et al., Phys. Rev. Lett. 45 (1980) 2081.

[11] M. Fierz, Helv. Phys. Acta 29 (1956) 128.

[12] P. Jordan, Nature 164 (1949) 637; Schwerkraft und Weltall (Vieweg, Braunschweig, 1955).

[13] T. Damour and G. Esposito-Farèse, Class. Quant. Grav. 9 (1992) 2093.

[14] P. Jordan, Z. Phys. 157 (1959) 112.

[15] C. Brans and R.H. Dicke, Phys. Rev. 124 (1961) 925.

[16] I.I. Shapiro, Phys. Rev. Lett. 13 (1964) 789.

[17] R.D. Reasenberg et al., Astrophys. J. 234 (1979) L219.

[18] D.S. Robertson, W.E. Carter and W.H. Dillinger, Nature 349 (1991) 768 ;

D.E. Lebach et al., Phys. Rev. Lett. 75 (1995) 1439.

[19] K. Nordtvedt, Phys. Rev. 170 (1968) 1186.

[20] I.I. Shapiro, in General Relativity and Gravitation 1989, ed. N. Ashby, D.F. Bartlett and W. Wyss (Cambridge University Press, Cambridge, 1990), 313-330.

[21] T. Damour and G. Esposito-Farèse, Phys. Rev. D 53 (1996) 5541. 
[22] R.A. Hulse and J.H. Taylor, Astrophys. J. Lett. 195 (1975) L51; see also the 1993 Nobel lectures in physics of Hulse (pp. 699-710) and Taylor (pp. 711-719) in Rev. Mod. Phys. 66, n03 (1994).

[23] P.S. Laplace, Traité de Mécanique Céleste, (Courcier, Paris, 17981825), Second part : book 10, chapter 7.

[24] T. Damour and N. Deruelle, Phys. Lett. A 87 (1981) 81;

T. Damour, C.R. Acad. Sci. Paris 294 (1982) 1335;

T. Damour, in Gravitational Radiation, eds N. Deruelle and T. Piran (North-Holland, Amsterdam, 1983) pp 59-144.

[25] J.H. Taylor, Class. Quant. Grav. 10 (1993) S167 (Supplement 1993) and references therein; see also J.H. Taylor's Nobel lecture quoted in [22].

[26] T. Damour and G. Esposito-Farèse, Phys. Rev. Lett. 70 (1993) 2220 .

[27] T. Damour and J.H. Taylor, Phys. Rev. D. 45 (1992) 1840.

[28] T. Damour and N. Deruelle, Ann. Inst. H. Poincaré 43 (1985) 107 and 44 (1986) 263.

[29] T. Damour and G. Esposito-Farèse, Phys. Rev. D 54 (1996) 1474.

[30] T. Damour and J.H. Taylor, Astrophys. J. 366 (1991) 501.

[31] J.H. Taylor, A. Wolszczan, T. Damour and J.M. Weisberg, Nature 355 (1992) 132.

[32] A. Wolszczan, Nature 350 (1991) 688.

[33] Z. Arzoumanian, Ph. D. thesis, Princeton University, 1995.

[34] T. Damour and D. Vokrouhlicky, Phys. Rev. D 53 (1996) 4177.

[35] A. Wolszczan and J.H. Taylor, to be published (quoted in Taylor's Nobel lecture [15]).

[36] C.M. Will and H.W. Zaglauer, Astrophys. J. 346 (1989) 366. 
[37] T. Damour and G. Schäfer, Phys. Rev. Lett. 66 (1991) 2549.

[38] T. Damour and D. Vokrouhlicky, Phys. Rev. D 53 (1996) 4177.

[39] R.D. Blandford et al. editors, Pulsars as physics laboratories, Phil. Trans. R. Soc. London A 341 (1992) pp 1-192; see notably the contributions by J.H. Taylor (pp. 117-134) and by T. Damour (pp. 135-149).

[40] J. Scherk and J.H. Schwarz, Nucl. Phys. B 81 (1974) 118; Phys. Lett. B 52 (1974) 347.

[41] B. de Carlos, J.A. Casas, F. Quevedo and E. Roulet, Phys. Lett. B 318 (1993) 447.

[42] R. Brustein and P.J. Steinhardt, Phys. Lett. B302 (1993) 196.

[43] G.D. Coughlan et al., Phys. Lett. B131 (1983) 59;

J. Ellis, D.V. Nanopoulos and M. Quiros, Phys. Lett. B174 (1986) 176 ;

T. Banks, D.B. Kaplan and A.E. Nelson, Phys. Rev. D49 (1994) 779 .

[44] T. Damour and A. Vilenkin, gr-qc/9610005, submitted to Phys. Rev. Lett.

[45] L. Randall and S. Thomas, Nucl. Phys. B 449 (1995) 229.

[46] D.H. Lyth and E.D. Stewart, Phys. Rev. Lett. 75 (1995) 201; Phys. Rev. D 53 (1996) 1784.

[47] T. Damour and A.M. Polyakov, Nucl. Phys. B 423 (1994) 532; Gen. Rel. Grav. 26 (1994), 1171.

[48] T. Damour and K. Nordtvedt, Phys. Rev. Lett. 70 (1993) 2217; Phys. Rev. D 48 (1993) 3436.

[49] T. Damour and A. Vilenkin, Phys. Rev. D53 (1996) 2981. 
[50] P.W. Worden, in Near Zero : New Frontiers of Physics, eds J.D. Fairbank et al. (Freeman, San Francisco, 1988) p. 766;

J.P. Blaser et al., STEP, Report on the Phase A Study, ESA document SCI (96)5, March 1996;

GEOSTEP Project, CNES report DPI/SC/FJC-N096/058, April 1996 ;

Fundamental Physics in Space, special issue of Class. Quant. Grav. 13 (1996).

MiniSTEP, NASA-ESA report, December 1996 (second issue). 